\title{
rTMS alleviates AD-induced cognitive impairment by inhibitng apoptosis in SAMP8 mouse
}

\author{
Zheng Bao ${ }^{1,2}$, Li Bao ${ }^{4}$, Na Han², Yueyun $\mathrm{Hou}^{2}$, Fumin Feng ${ }^{1,3}$ \\ ${ }^{1}$ School of Public Health, North China University of Science and Technology, Tangshan 063210, Hebei Province, \\ P.R. China \\ ${ }^{2}$ Child Health Division, Tongzhou Maternal and Child Health Hospital of Beijing, Beijing 101101, P.R. China \\ ${ }^{3}$ College of Life Science, North China University of Science and Technology, Tangshan 063210, Hebei, P.R. China \\ ${ }^{4}$ Langfang Guangyang District People's Hospital, Langfang 065099, Hebei, P.R. China
}

Correspondence to: Fumin Feng; email: hblgffm@126.com, https://orcid.org/0000-0002-3778-8968

Keywords: Alzheimer's disease, cognitive impairment, rTMS, apoptosis, cAMP/PKA/CREB signaling pathway

Abbreviations: rTMS: repetitive transcranial magnetic stimulation; MWM: Morris water maze; HE: hematoxylin-eosin;

TUNEL: terminal-deoxynucleotidyl transferase-mediated nick end labelling

Received: July 5, $2021 \quad$ Accepted: December 3, $2021 \quad$ Published: December 29, 2021

Copyright: (C) 2021 Bao et al. This is an open access article distributed under the terms of the Creative Commons Attribution License (CC BY 3.0), which permits unrestricted use, distribution, and reproduction in any medium, provided the original author and source are credited.

\section{ABSTRACT}

This study sought to investigate whether repetitive transcranial magnetic stimulation (rTMS) could alleviate cognitive dysfunction in SAMP8 mice by reducing cell apoptosis and activating the CAMP/PKA/CREB signalling pathway. A total of 40 SAMP8 mice were randomly assigned to the SAMP8 group ( $n=20)$, and rTMS treatment group (rTMS+SAMP8, $n=20$ ); additionally, 20 homologous and normal aged SAMR1 mice were used as the control group(n=20). The Morris water maze and $Y$ maze tests were applied to evaluate spatial learning and memory ability. Haematoxylin and eosin (HE) staining and terminal-deoxynucleotidyl transferase-mediated nick end labelling (TUNEL) were used to observe the changes in neurons in the cortex and hippocampus. Western blotting and RT-PCR were used to detect signalling related proteins. rTMS significantly improved spatial learning and memory deficits and morphological abnormalities in the hippocampus region of the hippocampus. In addition, rTMS reduced apoptosis of neurons caused by $A D$ and the expression of pro-apoptotic proteins (Caspase-3 and Bax) and increased the expression of an antiapoptotic protein (Bcl-2). Furthermore, rTMS activated the CAMP/PKA/CREB signalling pathway. These results showed that $r$ TMS could ameliorate cognitive deficits in AD mice by inhibiting apoptosis via activation the CAMP/PKA/CREB signalling pathway.

\section{INTRODUCTION}

As the life expectancy of the Chinese population increases, the number of patients with Alzheimer's disease will increase [1, 2]. At present, in developed countries, with the rapid ageing of the population, AD, together with heart disease, cancer and cerebrovascular disease, have become major diseases threatening the health and life of the elderly [3]. The incidence of AD is on the rise, bringing a large burden to patients, their families and society [4]. China's ageing population is developing rapidly. It is estimated that by 2050 , elderly population of $\mathrm{AD}$ patients in this country will reach 20 million. China is about to become the region with the largest number of $\mathrm{AD}$ patients and the fastest growth rate in the world. Thus, $\mathrm{AD}$ may become a major disease threatening the health of the elderly population in China [2]. Therefore, the strengthening of $\mathrm{AD}$ research not only has great significance for China, but also will have a profound historical impact. $\mathrm{AD}$ is the most common form of dementia and is characterized by loss of memory, language and behaviour abilities [5]. It has been reported that $\mathrm{AD}$ accounts for up to $80 \%$ of all types of dementia [6]. AD is a manifestation of brain ageing, and the pathology is extremely complex [7]. The structural changes include brain weight and volume 
reduction, neuronal volume reduction, neuronal selective loss, and neuronal protrusion reduction [8,9]. A large amount of evidence has shown that apoptosis is the ultimate fate of nerve cells in many neurodegenerative diseases, including $\mathrm{AD}$, and its excessive apoptosis is one of the main causes of $\mathrm{AD}[10,11]$. At present, there is no effective method to combat $\mathrm{AD}$, so the prevention and treatment of $\mathrm{AD}$ has become a focus of neuroscience research.

Repetitive transcranial magnetic stimulation (rTMS) is a non-invasive, electrodeless brain stimulation technique that uses a time-varying magnetic field to generate induced currents to directly stimulate cortical neurons and adjust the function of the latter [12]. rTMS can affect the excitability of the central nervous system by adjusting its frequency, intensity, stimulation interval and duration. For more than 20 years, rTMS has been widely used in various fields such as nerves, spirit, and psychology [13], and has made significant contributions to the understanding of perception, attention, consciousness, cortical function connections and plasticity. At present, the phenomenon of social ageing is very serious. Dementia and cognitive dysfunction are among the research hotspots of the $21 \mathrm{st}$ century. Future rTMS research should mainly focus on the mechanistic research and treatment effects of neuropsychiatric diseases, such as degenerative diseases of the nervous system $[14,15]$. The common symptoms of learning and memory decline are treatment effects. This study explored rTMS to observe how highfrequency rTMS affects the cognitive function of $\mathrm{AD}$, to provide a certain basis for the clinical prevention and treatment of AD.

\section{RESULTS}

\section{Improvement in cognitive function of $\mathrm{AD}$ mice after rTMS treatment}

To exclude the effects of rTMS on mice with cognitive deficits, MWM test was performed at 1-5 days after rTMS treatment in the three groups. In the orientation navigation experiment, we found significant differences in latency time between the Con group and P8 group, and the latency time of the $\mathrm{P} 8$ group was much longer than that of the Con group. However, the latency time was significantly decreased in the rTMS group compared with the P8 group (Figure 1A). In the spatial probe test, compared with the Con group, the platform crossings of the $\mathrm{P} 8$ group in the target quadrant were shortened, while rTMS prolonged the crossings of P8 mice in the platform quadrant (Figure 1B). However, no difference in swimming speed was observed between the three groups (Figure 1C). The Y-maze test was performed to assess the cognitive function of all animals.
Compared with the Con group, the number of training required to reach the $9 / 10$ standard in the P8 group was significantly increased, and the learning and memory performance was significantly decreased; the learning and memory scores of the rTMS group were significantly improved compared with those of the $\mathrm{p} 8$ group (Figure 1D, 1E). The results showed that rTMS improved cognitive disorders of in Alzheimer's disease which improved the performance of the mice.

\section{Reduction in the apoptosis of cortical and hippocampal neurons of P8 mice after rTMS treatment}

Morphological changes in cortical and hippocampal neurons were observed by HE staining. As shown in the HE staining results (Figure 2A), cortical and hippocampal neurons in the Con group were arranged in an orderly manner, chromatin was evenly distributed and nucleoli were clearly visible. In the P8 group, we observed a completely opposite pattern to the Con group. After rTMS treatment, the levels of neuron degeneration, necrosis, and loss were reduced compared with those in the P8 group. Then, TUNEL staining was used to observe the effect of rTMS on the apoptosis of cortical and hippocampal cells. As shown in Figure 2B, 2C, a small number of cortical and hippocampal neurons in the control group suffered apoptosis. In the P8 group, disordered neuronal cell arrangement was observed and the number of apoptotic cortical and hippocampal neurons increased. After rTMS treatment, the apoptotic neurons of P8 mice were significantly reduced. The results indicated that the improvement of learning and memory impairment by rTMS may be related to the reduction of neuron loss.

\section{Changes in the expression levels of apoptosis-related proteins and mRAN in the P8 mouse cortex and hippocampus after rTMS treatment}

Caspase-3, Bcl-2, and Bax expression levels were observed in 5 cortical and hippocampal tissue specimens by immunohistochemistry and qRT-PCR. Immunohistochemistry analysis of Caspase-3, Bcl-2, and Bax deposition demonstrated that in the cortex and hippocampus of P8 mice, the expression levels of Caspase- 3 and Bax were significantly increased, while the expression of Bcl-2 was significantly decreased. After rTMS treatment, the expression of Caspase- 3 and Bax decreased significantly, while the expression of Bcl-2 protein increased significantly (Figure 3). These findings indicated that P8 mice showed severe pathological features, significantly increased expression of apoptosis-related proteins (Caspase- 3 and Bax) and decreased expression of an anti-apoptosis-related protein (Bcl-2) in the cortex and hippocampus, 
fortunately rTMS reversed this pathological change. Furthermore, qRT-PCR results showed that compared with the control group, Caspase-3 and Bax mRNA expression was significantly increased while the Bcl-2 mRNA expression level was decreased, and these trends were significantly reversed by rTMS treatment
(Figure 4). The results indicated that the overexpression of proapoptotic proteins and the decrease in antiapoptotic proteins may be related to the cognitive function of P8 mice. rTMS could improve cognitive function by regulating the expression of these proteins and mRNAs.
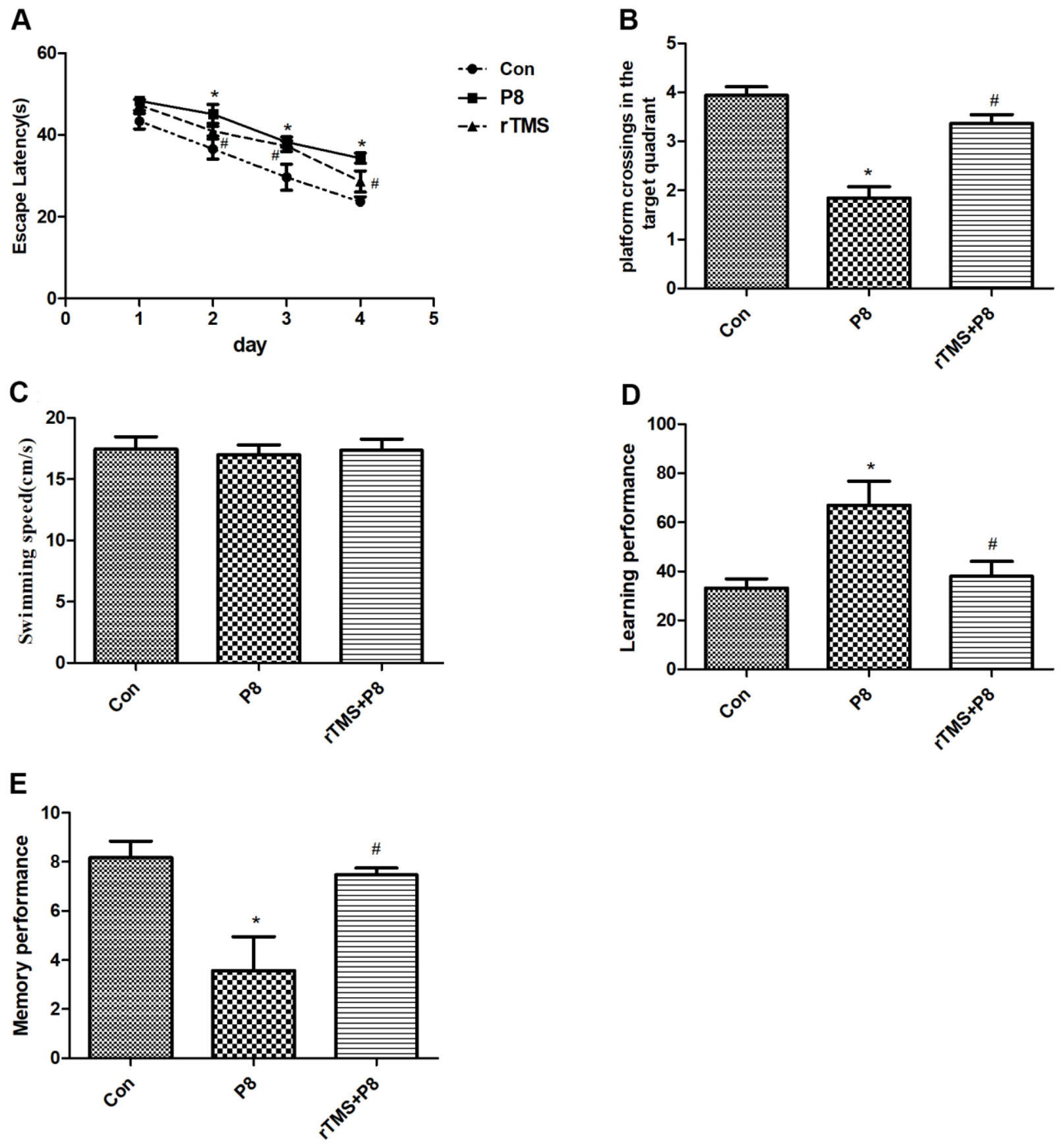

Figure 1. Improvement in cognitive function of the mice after rTMS treatment. (A) The latency time was compared between the three groups. (B) The dwell time in the target quadrant of mice in the three groups was compared. (C) The mean swimming speed of mice in three groups is shown. (D) The learning performance of mice in the three groups was compared. (E) The memory performance of mice in the three groups was compared. The data is demonstrated as the mean \pm standard deviation $(n=20) .{ }^{*} P<0.05$ vs. Con group; ${ }^{\#} P<0.05$ vs. P8 group. 
Involvement of the cAMP/PKA/CREB pathway in rTMS-mediated inhibition of neuronal apoptosis

To further determine whether the cAMP/PKA/CREB pathway participates in rTMS-related action, the expression of cAMP, PKAc, and CREB in mouse cortical and hippocampal tissue was determined by western blot. As shown in Figure 5, AD significantly decreased the cAMP, PKAc, and p-CREB expression, whereas rTMS increased the expression of cAMP,
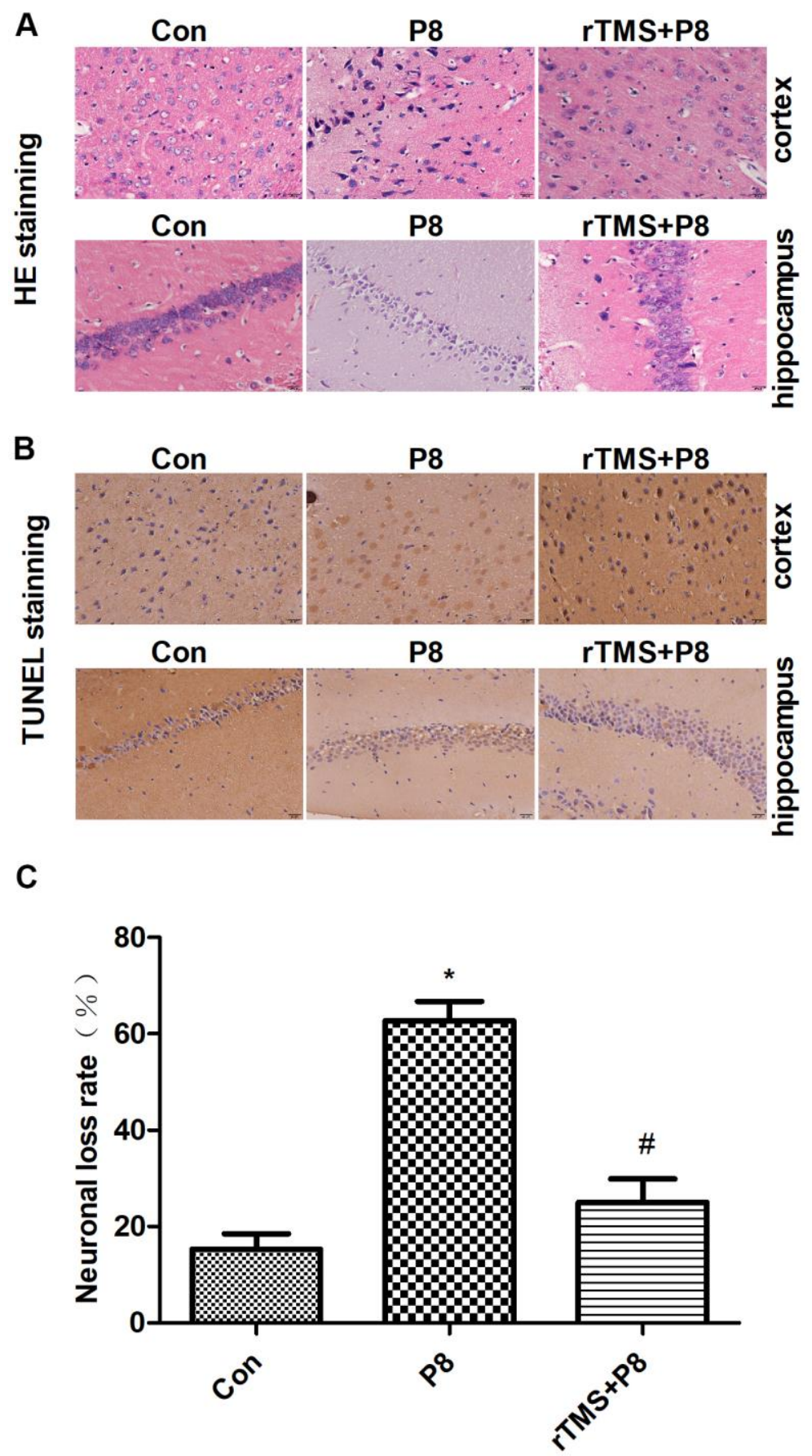

Figure 2. Reduction in neuronal loss in the cortical and hippocampal neurons of P8 mice after rTMS treatment. (A) The changes in the morphology of cortical and hippocampal neurons in mice were evaluated by HE staining (scale bar, 20 $\mu \mathrm{m}$ ). (B) TUNEL staining was performed for the mouse cortical and hippocampal regions (scale bar, 20 $\mu \mathrm{m}$, magnification, 10x eye piece x 40x objective). (C) Histogram analysis of TUNEL-positive cells. The data is demonstrated as the mean \pm standard deviation $(n=5) .{ }^{*} P<0.05$ vs. Con group; \# $P<0.05$ vs. P8 group. TUNEL, the terminal deoxynucleotidyl transferase nick-labelling; $\mathrm{HE}$, haematoxylin and eosin. 

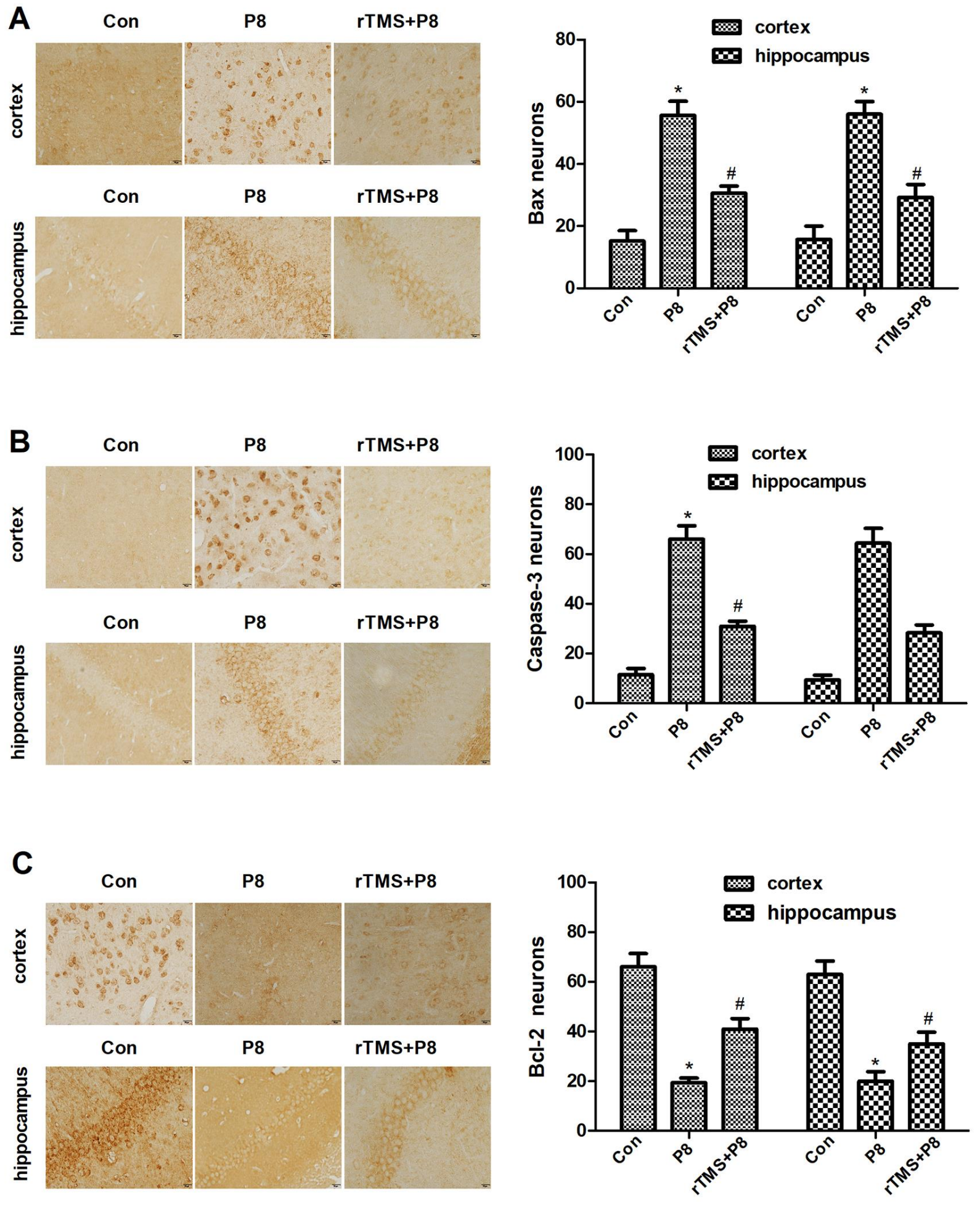

Figure 3. Changes in the expression levels of apoptosis-related proteins in cortical and hippocampal regions in the three groups after rTMS treatment. (A) Bax expression in the three groups was examined by immunohistochemistry staining and quantitative histogram analysis. (B) Caspase-3 expression in the three groups was examined by immunohistochemistry staining in three groups and quantitative histogram analysis. (C) Bcl-2 expression- in the three groups was examined by immunohistochemistry staining and quantitative histogram analysis. The data are presented as the mean \pm standard deviation $(n=5)$. ${ }^{*} P<0.05$ vs. Con group; \# $P<0.05$ vs. P8 group. 
PKAc, and p-CREB. Taken together, these results demonstrated that the cAMP/PKA/CREB pathway might be involved in the rTMS-induced changes in cortical and hippocampal tissue.

\section{DISCUSSION}

Several studies have shown that rTMS has a protective effect on the body, but the specific protective mechanism is not very clear [16]. Some studies have shown that rTMS may upregulate the expression of some protective mechanisms in the body, and simultaneously inhibit pro-cell injury mechanisms [17]. As an important second messenger in cells, cAMP plays an important role in the nervous system. Studies have confirmed that the cAMP/PKA/CREB signalling pathway is an important regulatory mechanism of the nervous system. The regulation of neuronal survival and axonal growth plays an important role, and may promote injured axon regeneration by inhibiting RhoA signalling pathways. Studies have shown that cAMP/PKA pathways are involved in mediating anti-inflammatory
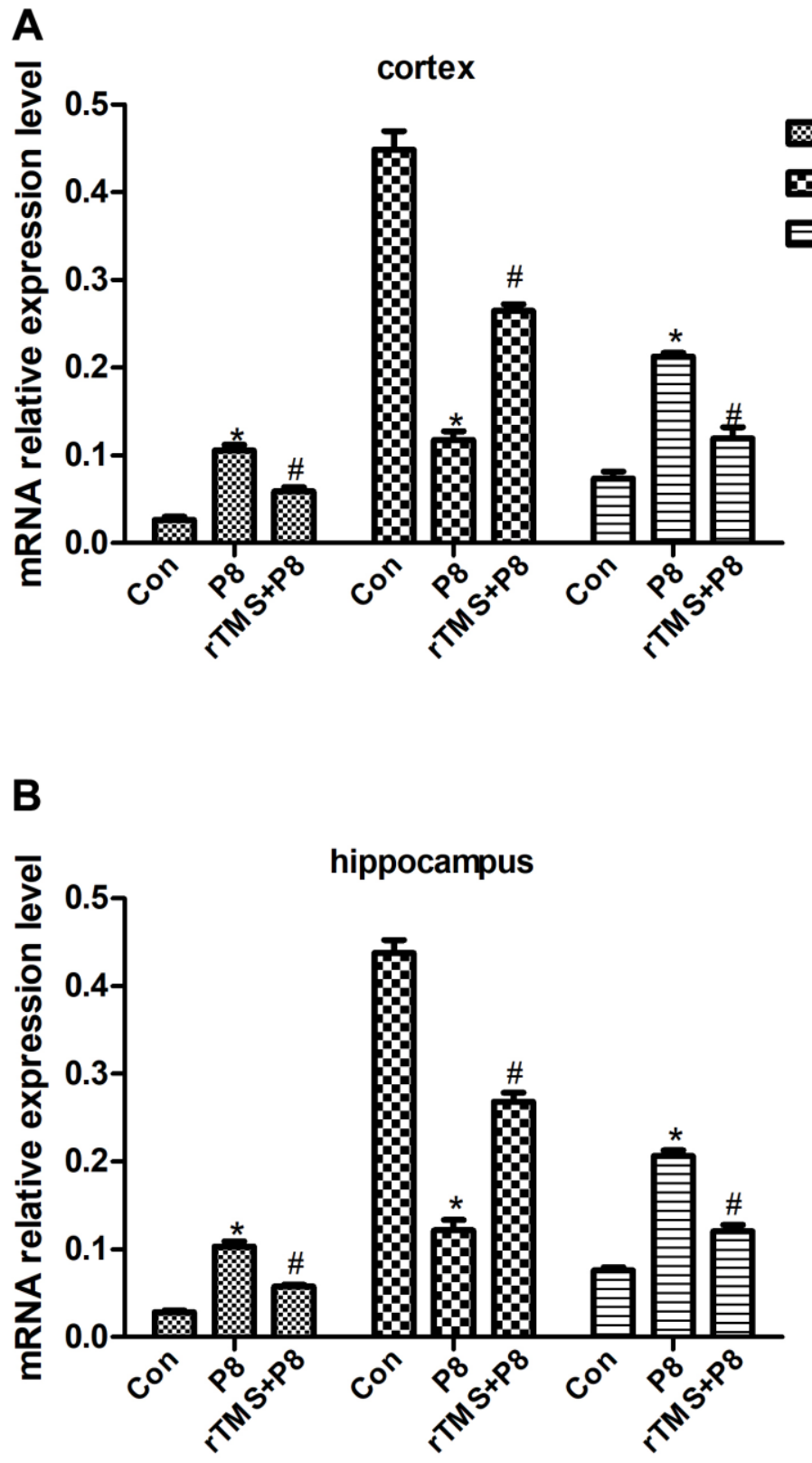

mRNA-Caspase-3

mex mRA-Bcl-2

$\Longrightarrow$ mRNA-Bax 
effects, and that inhibiting the cAMP/PKA pathways can aggravate mitochondrial metabolic disorders, leading to energy shortages and neuronal apoptosis [17-20].

rTMS is a magnetic stimulation method that has attracted the most attention in clinical medicine and neuroscience. Cotelli and Manenti et al. [21] used highfrequency rTMS to treat the dorsolateral prefrontal cortex in patients with $\mathrm{AD}$ and found that patients' naming ability was improved. In addition, high frequency rTMS has been found to have facilitate neurons that stimulate cortical nerve excitation [22]. The results of this study are consistent with this result, and the comparison between the P8 group and the control group after treatment was statistically significant, indicating that rTMS can significantly improve the learning and memory ability of AD. The effect of rTMS on the neural activity of learning and memory is a point at issue in brain science.

Studies have found that obvious apoptosis features in the hippocampus and cortex of $\mathrm{AD}$ mice, and the specific nerve tissues of patients with neurodegenerative diseases exhibit morphological changes of consistent with apoptosis [23, 24]. This evidence indicates that apoptosis is involved in the pathological process of $\mathrm{AD}$. Thus, rTMS may inhibit neuronal cell apoptosis in the cortex and hippocampus of mouse brain tissue. Caspase
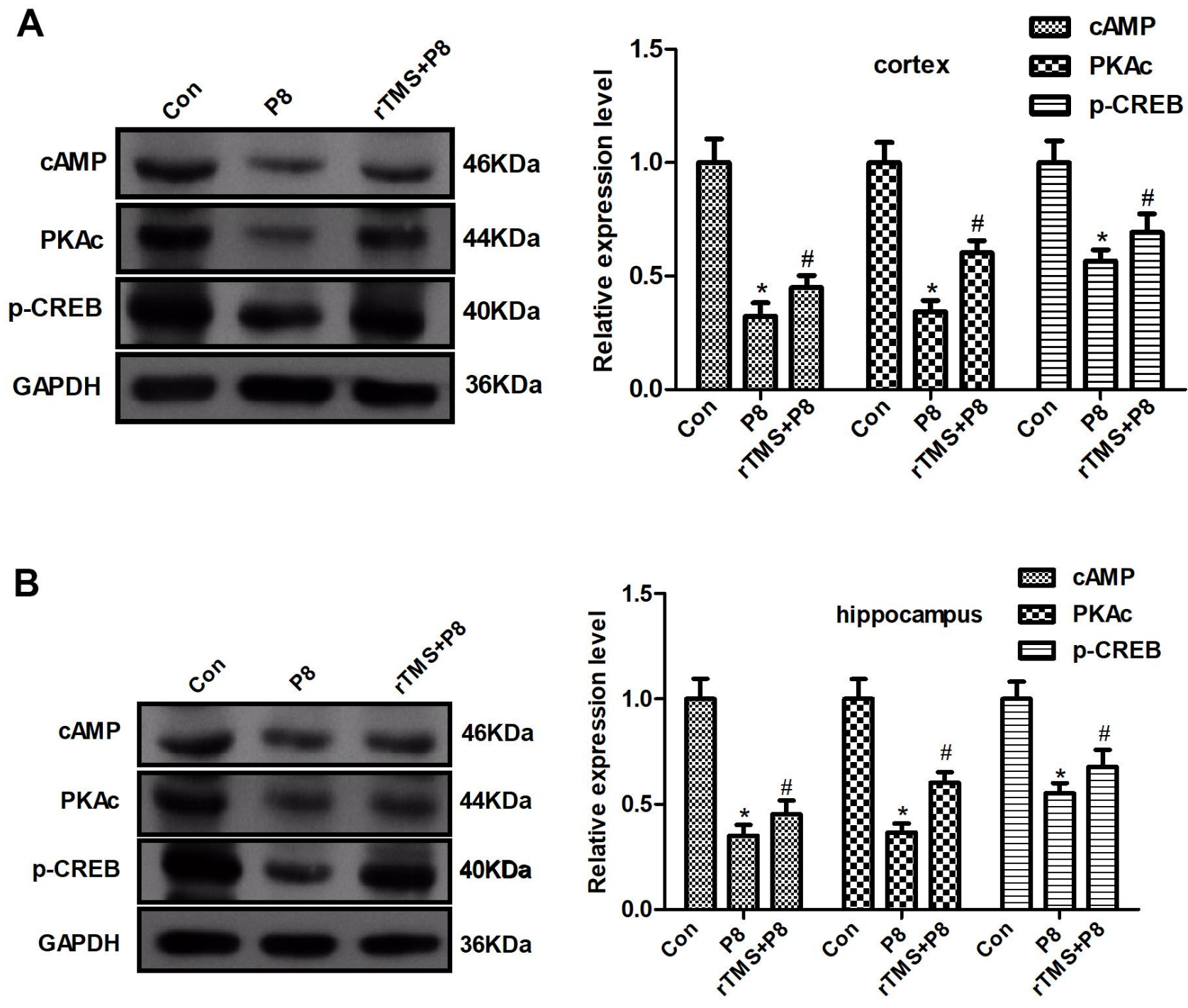

Figure 5. Changes in the CAMP/PKA/CREB pathway in P8 mouse cortex and hippocampus after rTMS treatment. (A) The expression of CAMP, PKAc and $p$ - CREB in the mouse cortex was determined by western blot and quantitative analysis of cAMP, PKAc and $p$ CREB expression. (B) The expression of CAMP, PKAc and $p$ - CREB in the mouse hippocampus was determined by western blot and the quantitative analysis of $C A M P$, PKAC and $p$ - CREB expression. The data are presented as the mean \pm standard deviation $(n=5)$. ${ }^{*} P<0.05$ vs. Con group; \# $\mathrm{P}<0.05$ vs. $\mathrm{P} 8$ group. 
is a specific apoptosis signal transduction molecule, and activated Caspase is the executor of apoptosis [25]. The activation of Caspases is manifested as a "waterfall" cascade reaction, and Caspase- 3 is the most critical apoptotic executive protease that descends in the Caspase cascade reaction, and plays the final pivotal role in the apoptosis initiated by various programs [26]. This experiment indicated that the expression of Caspase- 3 in the cerebral cortex and hippocampus of mice was significantly increased, which provided more substrates for Caspase- 3 and activated the occurrence of the Caspase cascade. rTMS significantly reduce the expression of Caspase-3, and inhibited the initiation of apoptosis. In the apoptosis of nerve cells, the Bcl-2 family is indispensable [27]. Bcl-2 not only acts upstream of Caspase-3, but is also the direct substrate of Caspase-3, and both play a key role in apoptotic events [28]. Studies have shown that Bcl-2 can promote the cell cycle and cell proliferation, and Bax gene overexpression can accelerate cell apoptosis and counteract the inhibitory effect of Bcl-2 on cell apoptosis $[29,30]$. The findings suggested that the expression of Bax in the cortex and hippocampus of $\mathrm{AD}$ model mice was significantly increased, while the expression of Bcl-2 was significantly reduced, but rTMS could significantly improve this change. The results of this experiment showed that apoptotic neurons increased, Bcl-2 decreased and Bax increased, in the cortex and hippocampus of AD model mice, indicating that Alzheimer's disease brain has a pro-apoptotic effect on tissues, and rTMS has an anti-apoptotic effect. This is in full agreement with the above Caspase- 3 results.

As one of the important second messengers in the cell, cAMP plays an important role in the nervous system. Studies have shown that the cAMP/PKA/CREB signalling pathway is one of the important regulatory mechanisms of the nervous system, which regulates neuron survival and axon growth [19]. It may play an important role in inhibiting the RhoA signalling pathway to promote the regeneration of damaged axons. Inhibition of the cAMP/PKA pathway will aggravate mitochondrial metabolism disorders and lead to energy deficiency and neuronal apoptosis [20]. This study found that the Bcl-2 protein in the cerebral cortex and hippocampus of $\mathrm{AD}$ mice decreased, while the expression of Bax protein and Caspase 3 protein increased. Morphological results showed that the cortex and hippocampal nerve cells were damaged. At the same time, cAMP and catalytic subunits were present in the cerebral cortex and hippocampus tissue. The expression of PKAc and p-CREB protein decreased, suggesting that the neurotoxicity of the cortex and hippocampus of $\mathrm{AD}$ mice may be related to the inhibition of the cAMP/ PKA/CREB signalling pathway, further downregulate the anti-apoptotic gene $\mathrm{Bcl}-2$ and upregulate the pro-apoptotic gene Bax, and then promote apoptosis. After rTMS treatment, morphological results and apoptosis-related proteins showed that the toxicity of nerve cells was reduced. At the same time, rTMS treatment also improved the inhibitory effect of $\mathrm{AD}$ mice in activating cAMP/PKA/CREB signalling pathway.

In our study, SAMP8 mice were used as the AD model. SAMP was selected by Japanese scholars for the breeding of natural AKP mutant mice and inbred and prolonged culture. SAMP consists of 9 sublines, among which SAMP8 is widely recognized as an ageing model of AD. SAMP8 has both the characteristics of natural ageing mice and learning and memory disorders similar to pathological changes of AD imagination. However, SAMP8 is considered unsuitable for long-term experiments because of its high cost and the short life span of the mice. In our study, apoptosis was not the primary reason of AD. However, in the present study, we aimed to investigate the effect of rTMS on cognition by inhibiting neuronal apoptosis. It is widely believed that the deposition of $A \beta$ in the brain is the central link in the pathological changes of $\mathrm{AD}$, which can trigger a series of pathological processes, that further promote the deposition of $A \beta$, thus forming a cascade reaction. Some studies have shown that $A \beta$ has neurotoxicity, which can lead to nerve cell apoptosis, thus impairing learning and memory function [31, 32]. Therefore, by inhibiting neuronal apoptosis, memory decline may be delayed.

\section{CONCLUSIONS}

The increase of in Bax levels, the decrease in Bcl-2 levels and the activation of downstream Caspase- 3 in AD mice induced the apoptosis of cortical and hippocampal nerve cells. rTMS could improve the changes of $\mathrm{Bax}$ and $\mathrm{Bcl}-2$ and the activation of downstream Caspase-3, promote neuroprotection, and activate the cAMP/PKA/CREB signalling pathway. One of the mechanisms by which rTMS reverses the neurotoxicity of Alzheimer's disease in mice may be by reversing the downregulation of the cAMP/PKA/CREB signalling pathway, which may be related to the mitochondrial energy metabolism of nerve cells. This phenomenon is worthy of further research.

\section{MATERIALS AND METHODS}

\section{Experimental animal and modeling}

Male 6-month-old 50 SAMP8 and 20 SAMR1 mice $(30 \pm 2 \mathrm{~g})$ were provided by Experimental Animal Center of North China University of Science and Technology and adaptively housed in the animal center for one week. The mice were housed separately under an 
alternative $12 \mathrm{~h}$ day/night cycle. The temperature and humidity in the were cab kept in a certain range ( $23 \pm$ $2^{\circ} \mathrm{C}$ and $50-65 \%$, respectively). Before the experiment, all mice were fasted for 12 to 16 hours, with free water intake. In fact, 10 mice were excluded from this study, and 60 qualified mice were used in the following study (excluding those with visual impairment, motor impairment and body mass). The study was approved by the Ethics Committee of North China University of Science and Technology.

\section{Grouping}

A total of 60 mice were randomly divided into three groups: the control group (Con, $n=20$ ), SAMP8 group $(\mathrm{P} 8, \mathrm{n}=20)$, and the rTMS+SAMP8 group (rTMS+P8, $\mathrm{n}=20$ ). The rTMS+P8 group received 10 groups of rTMS with a frequency of $5 \mathrm{~Hz}$ on the top of the skull every day, with an interval of $20 \mathrm{~s}$ between each group, a total of 1000 stimulation pulses, and a stimulation intensity of $1.2 \mathrm{~T}$, for 14 consecutive days. All studies followed the blind principle and after behavioral and histological analysis was completed, the animal codes were revealed.

\section{Morris water maze test}

The pool was a round stainless steel sink with a diameter of $90 \mathrm{~cm}$ (with a black platform submerged 2 $\mathrm{cm}$ under the surface, diameter: $10 \mathrm{~cm}$; height: $28 \mathrm{~cm}$ ), and the tank was filled with water $\left(22-25^{\circ} \mathrm{C}\right)$ to a depth of $30 \mathrm{~cm}$. The test was conducted for 5 days after 14 consecutive days treatment. The tank was randomly divided into 4 quadrants of EN, EW, SW and SN. The mice were placed into the selected pool position, and the swimming time and trajectory of the mice were recorded. The mice were given 60 s to find and climb on the platform stage. If the animal did not find the platform within $60 \mathrm{~s}$, the experimenter led the animals to the platform. The time it takes for the animals to find the platform is called the "escape latency". The first day was for training, with one training in the morning and one in the afternoon. The test was conducted 4 days after the end of treatment, and each mouse was tested four times a day for four consecutive days. The swimming speed was evaluated on the last day of the test, when the platform was removed. The average escape delay for a total of four trials was calculated.

\section{Y maze test}

The training was carried out in a three-part radial Y maze. The top of each arm was equipped with a 15-watt light bulb, and the bottom of the box was a parallel copper rod. The bottoms of the two dark arm boxes were energized, and lights illuminate the safe area. The mice were placed into the $\mathrm{Y}$ maze to adapt for $5 \mathrm{~min}$ before applying electrical stimulation (the stimulation parameters were set as: voltage $60 \mathrm{~V}$, and delay $5 \mathrm{~s}$ ). During training, the safe zone is randomly changed, with the correct response of the mouse to escape to the safe zone was after receiving an electric shock stipulated, and the mice were allowed to stay in the safe zone for $30 \mathrm{~s}$ to consolidate memory. When the rat had 9 correct responses (9/10) in 10 consecutive training sessions, this indicated a learning grid. The number of training required before the mouse reaches the $9 / 10$ standard is recorded to quantify the performance of the mouse when it was qualified. Memory ability test: $24 \mathrm{~h}$ after the training, the mice were trained 10 times according to the above method, and the number of correct responses was recorded as the memory score to represent the level of memory ability.

\section{Histopathological examinations}

After the MWM test, cortical and hippocampal tissues of mice in all three groups were removed immediately at 24 $\mathrm{h}$ after reperfusion. Then the mouse brain specimens fixed with $10 \%$ formaldehyde were conventionally dehydrated, soaked in wax, embedded and sectioned. The sections were stained with hematoxylin and eosin (HE) for histopathological examination to observe the morphological changes of neurons in an optical microscope under $40 x$ magnification(10x eye piece $x$ 40x objective) in the hippocampus of mice.

\section{Immunohistochemistry}

Cortex and hippocampal tissues were exposed to $4 \%$ paraformaldehyde at $4^{\circ} \mathrm{C}$ and $30 \%$ sucrose for 2 hours respectively, embedded in paraffin and sectioned at $5 \mu \mathrm{m}$ immunohistochemistry experiments. The slices were washed three times with $0.01 \mathrm{~mol} / \mathrm{L}$ TBS buffer, and incubated at room temperature for $1 \mathrm{~h}$ in blocking buffer. After that, the slices, at $4^{\circ} \mathrm{C}$, were incubated with primary antibodies including Caspase- 3 (Abcam, USA, ab184787,1:1000), Bax (Abcam, USA, ab32503,1:1000), and Bcl-2 (Abcam, USA, ab182858, 1:1000) overnight. Slices were washed three times for 10 min each in PBS at room temperature, and then incubated with HRP-labelled goat anti-mouse secondary antibody at $37^{\circ} \mathrm{C}$ for $30 \mathrm{~min}$. DAB chromogenic agent was used for colour development. Slices were rinsed thoroughly with tap water, re-stained, dehydrated, made transparent as necessary, and sealed. The positive cells were observed under a microscope. We used Image ProPlus software to do statistical analysis.

\section{TUNEL staining}

TUNEL (terminal deoxynucleotidyl transferase (TdT)mediated dUTP nick end labelling) staining was used to 
investigate the role of rTMS in neurons of cortex and hippocampus induced by AD. The mouse cortex and hippocampus specimens fixed with $10 \%$ formaldehyde were conventionally dehydrated, soaked in wax, embedded and sectioned, and cut into slices. The sections were deparaffinized in xylene, hydrated, digested with trypsin, and stained in TUNEL mix. Nest, the slices were dehydrated, made transparent, sealed with neutral gum. Finally, the sections were observed with an optical microscope and the apoptosis rate of cortical and hippocampal neurons was calculated.

\section{Quantitative real-time PCR analysis}

The expression levels of the mRNAs (Caspase-3, Bax, and Bcl-2) were quantified using qRT-PCR analysis. According to the manufacturer's instructions, the total RNA of the mouse cortex and hippocampus was isolated with TRIzol reagent, and then cDNA was obtained by reverse transcription. qRT-PCR was performed as previously described [33]. Calculations were measured using the $2-\Delta \Delta \mathrm{Ct}$ method to analyze the relative expression of each gene, and the bar chart was drawn using GraphPad Prism 5.0. The primers were as follows: Caspase-3: forward: 5'-CCGCTTATAACTGTTGCT GT-3', reverse: 5'-TTCCCAGCGGTCCGCTTCAT-3'; Bax: forward: 5'-CCCAGAGGCGGGGTTTCA-3', reverse: 5'-GGAAAAAGACCTCTCGGGGG-3'; Bcl-2: forward:5'-CATATCTGTTTCGAGAATCA-3', reverse: 5'-CACCCGTTTCTCCGATAAGCA-3'; $\beta$-actin: forward: 5'-CTTCGCTTTCGAACAT-3', reverse: 5'CCACATATTCCTCCAACTGACC-3'.

\section{Western blot}

The mouse cortex and hippocampal tissue were rapidly isolated on ice, total proteins were extracted, the protein concentration was determined by the $\mathrm{BCA}$ reagent total proteins were extracted and the concentration was determined by BCA reagent (Abcam, USA, ab146331). The samples were subjected to SDSPAGE electrophoresis, and the separated proteins were transferred to PVDF membranes. The blots were blocked with 5\% skim milk at room temperature for $1 \mathrm{~h}$, and then the membrane was incubated with the primary antibody overnight at $4^{\circ} \mathrm{C}$, including anti-cAMP (Abcam, USA, ab76238, 1:2000), anti-PKA (catalytic subunits) (Abcam, USA, ab59218, 1:1000), anti-CREB (Abcam, USA, ab32515, 1:500). Then, the cells were incubated with horseradish peroxidase conjugated goat anti-rabbit IgG H\&L (Abcam, USA, ab6721,1:5000) for $2 \mathrm{~h}$ at room temperature. After incubation with a properly titrated secondary antibody, the cells underwent ECL luminescence development. The developed image was scanned to a computer for analysis after exposure.

\section{Statistical analysis}

The data in the experiment are expressed as the mean \pm standard deviation (S.D.) and analysed by SPSS 20.0. There was a minimum of five mice per group. Oneway ANOVA was used to analyze the results followed by the Newman-Keuls post-hoc test. A P value of $<0.05$ was considered significant for all statistical analyses.

\section{Ethics statement}

The mouse experiments were approved by the Animal Ethics Welfare Committee of North China University of Science and Technology.

\section{AUTHOR CONTRIBUTIONS}

The study was designed by Fumin Feng; experiments were performed by Zheng Bao, Li Bao, Na Han, Yueyun Hou; data was analyzed and paper was written by Zheng Bao and Li Bao; the manuscript was revised by Fumin Feng. All authors reviewed and agreed with this manuscript.

\section{CONFLICTS OF INTEREST}

The authors declare that they have no conflicts of interest.

\section{FUNDING}

This work was supported by Hebei graduate innovation funding project (No: CXZZBS2019137).

\section{REFERENCES}

1. Chan KY, Wang W, Wu JJ, Liu L, Theodoratou E, Car J, Middleton L, Russ TC, Deary IJ, Campbell H, Wang W, Rudan I, and Global Health Epidemiology Reference Group (GHERG). Epidemiology of Alzheimer's disease and other forms of dementia in China, 1990-2010: a systematic review and analysis. Lancet. 2013; 381:2016-23.

https://doi.org/10.1016/S0140-6736(13)60221-4 PMID:23746902

2. Zhang $Y$, Li Y, Ma L. Recent advances in research on Alzheimer's disease in China. J Clin Neurosci. 2020; 81:43-6.

https://doi.org/10.1016/i.jocn.2020.09.018 PMID:33222956

3. Heron M. Deaths: Leading Causes for 2017. Natl Vital Stat Rep. 2019; 68:1-77.

PMID: $\underline{32501203}$

4. Kalaria RN, Maestre GE, Arizaga R, Friedland RP, Galasko D, Hall K, Luchsinger JA, Ogunniyi A, Perry EK, 
Potocnik F, Prince M, Stewart R, Wimo A, et al, and World Federation of Neurology Dementia Research Group. Alzheimer's disease and vascular dementia in developing countries: prevalence, management, and risk factors. Lancet Neurol. 2008; 7:812-26.

https://doi.org/10.1016/S1474-4422(08)70169-8

PMID:18667359

5. Jia L, Quan M, Fu Y, Zhao T, Li Y, Wei C, Tang Y, Qin Q, Wang F, Qiao Y, Shi S, Wang YJ, Du Y, et al, and Group for the Project of Dementia Situation in China. Dementia in China: epidemiology, clinical management, and research advances. Lancet Neurol. 2020; 19:81-92.

https://doi.org/10.1016/S1474-4422(19)30290-X PMID:31494009

6. Weller J, Budson A. Current understanding of Alzheimer's disease diagnosis and treatment. F1000Res. 2018; 7:F1000.

https://doi.org/10.12688/f1000research.14506.1 PMID:30135715

7. Crous-Bou M, Minguillón C, Gramunt N, Molinuevo JL. Alzheimer's disease prevention: from risk factors to early intervention. Alzheimers Res Ther. 2017; 9:71.

https://doi.org/10.1186/s13195-017-0297-z

PMID:28899416

8. Šimić G, Babić Leko M, Wray S, Harrington CR, Delalle I, Jovanov-Milošević $N$, Bažadona $D$, Buée $L$, de Silva R, Di Giovanni G, Wischik CM, Hof PR. Monoaminergic neuropathology in Alzheimer's disease. Prog Neurobiol. 2017; 151:101-38.

https://doi.org/10.1016/j.pneurobio.2016.04.001 PMID:27084356

9. Anderton $\mathrm{BH}$. Ageing of the brain. Mech Ageing Dev. 2002; 123:811-7. https://doi.org/10.1016/s0047-6374(01)00426-2 PMID:11869738

10. Robinson AA, Abraham CR, Rosene DL. Candidate molecular pathways of white matter vulnerability in the brain of normal aging rhesus monkeys. Geroscience. 2018; 40:31-47.

https://doi.org/10.1007/s11357-018-0006-2

PMID:29357021

11. Obulesu M, Lakshmi MJ. Apoptosis in Alzheimer's disease: an understanding of the physiology, pathology and therapeutic avenues. Neurochem Res. 2014; 39:2301-12.

https://doi.org/10.1007/s11064-014-1454-4

PMID:25322820

12. Behl C. Apoptosis and Alzheimer's disease. J Neural Transm (Vienna). 2000; 107:1325-44.

https://doi.org/10.1007/s007020070021

PMID:11145007
13. Kedia S. Complementary and Integrative Approaches for Pediatric Headache. Semin Pediatr Neurol. 2016; 23:44-52.

https://doi.org/10.1016/i.spen.2016.01.010 PMID:27017022

14. Pourzitaki C, Dardalas I, Poutoglidou F, Kouvelas D, Kimiskidis VK. The Combination of rTMS and Pharmacotherapy on In Vitro Models: A Mini-Review. CNS Neurol Disord Drug Targets. 2020; 19:220-6. https://doi.org/10.2174/187152731966620051810071 6 PMID: $\underline{32418533}$

15. Chen X, Chen S, Liang W, Ba F. Administration of Repetitive Transcranial Magnetic Stimulation Attenuates $A \beta_{1-42}$-Induced Alzheimer's Disease in Mice by Activating $\beta$-Catenin Signaling. Biomed Res Int. 2019; 2019:1431760.

https://doi.org/10.1155/2019/1431760 PMID:30949496

16. Nörz D, Fischer N, Schultze A, Kluge $S$, Mayer-Runge $U$, Aepfelbacher M, Pfefferle S, Lütgehetmann M. Clinical evaluation of a SARS-CoV-2 RT-PCR assay on a fully automated system for rapid on-demand testing in the hospital setting. J Clin Virol. 2020; 128:104390.

https://doi.org/10.1016/j.jcv.2020.104390 PMID:32388471

17. Taylor JL, Hambro BC, Strossman ND, Bhatt $P$, Hernandez B, Ashford JW, Cheng JJ, IV M, Adamson MM, Lazzeroni LC, McNerney MW. The effects of repetitive transcranial magnetic stimulation in older adults with mild cognitive impairment: a protocol for a randomized, controlled three-arm trial. BMC Neurol. 2019; 19:326.

https://doi.org/10.1186/s12883-019-1552-7 PMID:31842821

18. Zong X, Li Y, Liu C, Qi W, Han D, Tucker L, Dong Y, Hu S, Yan $X$, Zhang $Q$. Theta-burst transcranial magnetic stimulation promotes stroke recovery by vascular protection and neovascularization. Theranostics. 2020; 10:12090-110.

https://doi.org/10.7150/thno.51573

PMID:33204331

19. Wang $Y L$, Zhu $X L$, Sun $M H$, Dang YK. Effects of astaxanthin onaxonal regeneration via cAMP/PKA signaling pathway in mice with focal cerebral infarction. Eur Rev Med Pharmacol Sci. 2019; 23:135-43. https://doi.org/10.26355/eurrev_201908_18640 PMID:31389584

20. Qi YC, Duan GZ, Mao W, Liu Q, Zhang YL, Li PF. Taurochenodeoxycholic acid mediates cAMP-PKACREB signaling pathway. Chin J Nat Med. 2020; 18:898-906.

https://doi.org/10.1016/S1875-5364(20)60033-4 PMID:33357720 
21. Yang $Y$, Ma $S$, Wei $F$, Liang $G$, Yang $X$, Huang $Y$, Wang J, Zou Y. Pivotal role of CAMP-PKA-CREB signaling pathway in manganese-induced neurotoxicity in PC12 cells. Environ Toxicol. 2019; 34:1052-62.

https://doi.org/10.1002/tox.22776

PMID:31161640

22. Cotelli M, Manenti R, Alberici A, Brambilla M, Cosseddu M, Zanetti O, Miozzo A, Padovani A, Miniussi C, Borroni B. Prefrontal cortex rTMS enhances action naming in progressive non-fluent aphasia. Eur J Neurol. 2012; 19:1404-12.

https://doi.org/10.1111/i.1468-1331.2012.03699.x PMID:22435956

23. Lefaucheur JP. Transcranial magnetic stimulation. Handb Clin Neurol. 2019; 160:559-80. https://doi.org/10.1016/B978-0-444-64032-1.00037-0 PMID:31277876

24. Garvock-de Montbrun T, Fertan E, Stover K, Brown RE. Motor deficits in 16-month-old male and female 3xTgAD mice. Behav Brain Res. 2019; 356:305-13. https://doi.org/10.1016/i.bbr.2018.09.006 PMID:30208295

25. Patrick GN, Zukerberg L, Nikolic M, de la Monte S, Dikkes P, Tsai LH. Conversion of p35 to p25 deregulates Cdk5 activity and promotes neurodegeneration. Nature. 1999; 402:615-22. https://doi.org/10.1038/45159 PMID:10604467

26. Elinos-Báez CM, Maldonado V, Meléndez-Zajgla J. [Caspases: apoptosis inducing molecules]. Gac Med Mex. 2003; 139:493-9.

PMID:14635568

27. Han H, Teng W, Chen T, Zhao J, Jin Q, Qin Z, Ji J. A cascade enzymatic reaction activatable gemcitabine prodrug with an AIE-based intracellular light-up apoptotic probe for in situ self-therapeutic monitoring. Chem Commun (Camb). 2017; 53:9214-7. https://doi.org/10.1039/c7cc04872f PMID:28766646
28. Li M, Peng J, Song $Y$, Liang $H$, Mei $Y$, Fang $Y$. Electroacupuncture combined with transcranial magnetic stimulation improves learning and memory function of rats with cerebral infarction by inhibiting neuron cell apoptosis. J Huazhong Univ Sci Technolog Med Sci. 2012; 32:746-9.

https://doi.org/10.1007/s11596-012-1028-0

PMID:23073807

29. Renvoizé C, Roger R, Moulian N, Bertoglio J, Bréard J. $\mathrm{Bcl}-2$ expression in target cells leads to functional inhibition of caspase-3 protease family in human NK and lymphokine-activated killer cell granule-mediated apoptosis. J Immunol. 1997; 159:126-34.

PMID: 9200447

30. Vermeulen K, Berneman ZN, Van Bockstaele DR. Cell cycle and apoptosis. Cell Prolif. 2003; 36:165-75. https://doi.org/10.1046/i.1365-2184.2003.00267.x PMID:12814432

31. Karavias DD, Tsamandas AC, Tepetes K, Kritikos N, Kourelis T, Ravazoula P, Vagenas K, Siasos N, Mirra N, Bonikos DS. BCL-2 and BAX expression and cell proliferation, after partial hepatectomy with and without ischemia, on cholestatic liver in rats: an experimental study. J Surg Res. 2003; 110:399-408. https://doi.org/10.1016/s0022-4804(03)00003-9 PMID: 12788671

32. Rad SK, Arya A, Karimian H, Madhavan P, Rizwan F, Koshy S, Prabhu G. Mechanism involved in insulin resistance via accumulation of $\beta$-amyloid and neurofibrillary tangles: link between type 2 diabetes and Alzheimer's disease. Drug Des Devel Ther. 2018; 12:3999-4021.

https://doi.org/10.2147/DDDT.S173970

PMID: 30538427

33. Xu C, Xiao Z, Wu H, Zhou G, He D, Chang Y, Li Y, Wang $G$, Xie M. BDMC protects $A D$ in vitro via $A M P K$ and SIRT1. TransI Neurosci. 2020; 11:319-27. https://doi.org/10.1515/tnsci-2020-0140 PMID:33335771 